\title{
ON INFINITESIMAL TEICHMÜLLER SPACE
}

\author{
FAN JINHUA ${ }^{\bowtie}$ and CHEN JIXIU
}

(Received 22 January 2008)

\begin{abstract}
Much research has been done on the geometry of Teichmüller space and Hamilton sequences of extremal Beltrami differentials. This paper discusses some problems concerning infinitesimal Teichmüller geodesic discs and Hamilton sequences of extremal Beltrami differentials in the tangent space of an infinite-dimensional Teichmüller space.
\end{abstract}

2000 Mathematics subject classification: 30 C75.

Keywords and phrases: infinitesimal Teichmüller space, geodesic disc, Hamilton sequence.

\section{Introduction}

Let $R$ be a given Riemann surface of analytical infinite type. We denote by $M(R)$ the open unit ball in the space $L^{\infty}(R)$ of all essentially bounded Beltrami differentials on $R$. Let $A(R)$ be the space of integrable quadratic differentials $\varphi$ that are holomorphic on $R$, and let $A_{1}(R)$ be the unit sphere of $A(R)$. Two elements $\mu, v \in M(R)$ are infinitesimally equivalent, denoted by $\mu \approx \nu$, if $\int_{R} \mu \phi=\int_{R} \nu \phi$ for all $\phi \in A(R)$. This equivalence relation partitions $M(R)$ into equivalence classes, and the space of all equivalence classes is called infinitesimal Teichmüller space, denoted by $B(R)$. It is known that $B(R)$ is the tangent space of Teichmüller space $T(R)$ at the basepoint. For some basic definitions and notation relating to Teichmüller space, we refer the reader to the books $[4,5]$ and the paper [11].

Given $\mu \in M(R)$, we denote by $[\mu]_{B}$ the set of all elements $\nu \in M(R)$ which are infinitesimally equivalent to $\mu$, and we set

$$
\|\mu\|=\inf \left\{\|\nu\|_{\infty}: v \in[\mu]_{B}\right\} .
$$

The infinitesimal Teichmüller distance between two points $[\mu]_{B}$ and $[\nu]_{B}$ is defined as

$$
d_{B}\left([\mu]_{B},[\nu]_{B}\right)=\inf _{\eta \in[\mu-\nu]_{B}}\|\eta\|_{\infty} .
$$

This work was supported by the Chinese NNSF grant no. 10571028.

(c) 2008 Australian Mathematical Society 0004-9727/08 \$A2.00+0.00 
We say that $\mu$ is extremal in $B(R)$ if $\|\mu\|_{\infty}=\|\mu\|$. It is known that a Beltrami differential $\mu$ is extremal in $B(R)$ if and only if there is a sequence $\left\{\varphi_{n}\right\}$ in $A_{1}(R)$ such that

$$
\|\mu\|_{\infty}=\lim _{n \rightarrow \infty}\left|\int_{R} \mu \varphi_{n}\right|,
$$

and we call such $\left\{\varphi_{n}\right\}$ a Hamilton sequence for $\mu$. For any $\mu \in M(R)$, we can define the boundary seminorm $b([\mu])$ by

$$
b([\mu])=\inf \left\{\left\|\left.\nu\right|_{R \backslash F}\right\|_{\infty}: v \approx \mu, F \text { is a compact subset of } R\right\} .
$$

An infinitesimal equivalent class $[\mu]_{B}$ is called an infinitesimal Strebel point if $\|\mu\|>b([\mu])$; otherwise it is called an infinitesimal non-Strebel point.

\section{Infinitely many geodesic discs in $B(R)$}

By definition, a geodesic disc in $B(R)$ is the image of a map $\Gamma: \triangle \rightarrow B(R)$ which is isometric with respect to the Euclidean distance in $\triangle$ and the infinitesimal Teichmüller distance in $B(R)$, where $\triangle$ denotes the unit disc of the complex plane.

For any point $[\mu]_{B} \neq[0]_{B}$, there is at least one geodesic disc passing through $[0]_{B}$ and $[\mu]_{B}$. In fact, any extremal Beltrami differential $\mu$ contained in $[\mu]_{B}$ determines a map

$$
\Gamma_{\mu}: \Delta \rightarrow B(R), \quad t \mapsto\left[t \mu /\|\mu\|_{\infty}\right]_{B},
$$

and $\Gamma_{\mu}$ is a holomorphic isometry with $\Gamma_{\mu}(0)=[0]_{B}$ and $\Gamma_{\mu}\left(\|\mu\|_{\infty}\right)=[\mu]_{B}$ (see [9]).

For a given point $[\mu]_{B}$ in $B(R)$, we want to know how many geodesic discs there are which contain $[0]_{B}$ and $[\mu]_{B}$, as well as how many isometries $\Gamma: \triangle \rightarrow B(R)$ there are with $\Gamma_{\mu}(0)=[0]_{B}$ and $\Gamma_{\mu}\left(\|\mu\|_{\infty}\right)=[\mu]_{B}$.

From [9], we know that if $[\mu]_{B}$ contains an extremal differential $\mu$ such that $|\mu|$ is not a constant, then there are infinitely many holomorphic isometries and geodesic discs that possess the aforementioned properties. In this paper, we consider more general cases and establish the following theorems.

THEOREM 2.1. Let $[\mu]_{B} \neq[0]_{B}$ be an infinitesimal non-Strebel point in $B(R)$. Then there are infinitely many isometries $\Gamma: \Delta \rightarrow B(R)$ with $\Gamma(0)=[0]_{B}$ and $\Gamma\left(\|\mu\|_{\infty}\right)=[\mu]_{B}$.

THEOREM 2.2. Let $[\mu]_{B} \neq[0]_{B}$ be an infinitesimal non-Strebel point in $B(R)$. Then there are infinitely many geodesic discs in $B(R)$ containing $[0]_{B}$ and $[\mu]_{B}$.

REMARK 2.3. Theorems 2.1 and 2.2 correspond to the results in Teichmüller space that were obtained by $\mathrm{Li}$ [8]. Recently, however, we proved in [2] that there being only one geodesic joining $[0]_{B}$ and $[v]_{B}$ in $B(R)$ does not imply that there will be only one geodesic joining the $[0]_{T}$ and $[v]_{T}$ in $T(R)$, and vice versa. Therefore, our theorems cannot be deduced directly from the results in [8]. 
Let $[\mu]_{B}$ be an infinitesimal non-Strebel point as in Theorems 2.1 and 2.2. We construct the isometries and geodesic discs as follows.

Let $\mu$ be an extremal Beltrami differential contained in $[\mu]_{B}$, and let $k_{0}=\|\mu\|_{\infty}$; then $0<k_{0}<1$. Suppose $E$ is a compact subset of $R$, and define $\mu_{t}(z)$ with parameter $t \in \triangle$ by

$$
\mu_{t}(z):=\mu(z) / k_{0} \quad \text { for } z \in R,|t| \leq k_{0}
$$

and

$$
\mu_{t}(z)= \begin{cases}\mu(z) / k_{0} & \text { for } z \in R \backslash E,|t|>k_{0}, \\ \mu(z) /|t| & \text { for } z \in E,|t|>k_{0} .\end{cases}
$$

We then define the map

$$
\Gamma: \triangle \rightarrow B(R), \quad t \mapsto\left[t \mu_{t}(z)\right]_{B} .
$$

LEMMA 2.4. The map $\Gamma$ is an isometry.

Proof. (1) Suppose $\left|t_{1}\right| \leq k_{0}$ and $\left|t_{2}\right| \leq k_{0}$. In this case, by the definition of $\mu_{t}(z)$,

$$
\begin{aligned}
d_{B}\left(\left[t_{1} \mu_{t_{1}}\right]_{B},\left[t_{2} \mu_{t_{2}}\right]_{B}\right) & =d_{B}\left(\left[t_{1} \mu / k_{0}\right]_{B},\left[t_{2} \mu / k_{0}\right]_{B}\right) \\
& =\inf _{\eta \in\left[\left(\left(t_{1}-t_{2}\right) / k_{0}\right) \mu\right]_{B}}\|\eta\|_{\infty} \\
& =\left\|\frac{t_{1}-t_{2}}{k_{0}} \mu\right\|_{\infty}=\left|t_{1}-t_{2}\right| .
\end{aligned}
$$

(2) Suppose $\left|t_{1}\right| \geq k_{0}$ and $\left|t_{2}\right| \geq k_{0}$. In this case, by the definition of $\mu_{t}(z)$,

$$
d_{B}\left(\left[t_{1} \mu_{t_{1}}\right]_{B},\left[t_{2} \mu_{t_{2}}\right]_{B}\right)=\inf _{\eta \in\left[t_{1} \mu_{t_{1}}-t_{2} \mu_{t_{2}}\right]_{B}}\|\eta\|_{\infty}
$$

where

$$
t_{1} \mu_{t_{1}}-t_{2} \mu_{t_{2}}=\left\{\begin{array}{l}
\frac{t_{1}-t_{2}}{k_{0}} \mu(z) \quad \text { for } z \in R \backslash E, \\
\left(\frac{t_{1}}{\left|t_{1}\right|}-\frac{t_{2}}{\left|t_{2}\right|}\right) \mu(z) \quad \text { for } z \in E .
\end{array}\right.
$$

Let $t_{1}=\rho_{1} e^{\mathrm{i} \theta_{1}}$ and $t_{2}=\rho_{2} e^{\mathrm{i} \theta_{2}}$, where $\rho_{2} \geq \rho_{1} \geq k_{0}$. Set $\rho=\rho_{2} / \rho_{1} \geq 1$ and $e^{\mathrm{i} \theta}=$ $e^{\mathrm{i} \theta_{2}} / e^{\mathrm{i} \theta_{1}}$. By a simple computation, we obtain

$$
\begin{aligned}
\frac{\left|t_{1}-t_{2}\right|^{2}}{k_{0}^{2}} & \geq 1+\rho^{2}-2 \rho \cos \theta \\
\left|\frac{t_{1}}{\left|t_{1}\right|}-\frac{t_{2}}{\left|t_{2}\right|}\right|^{2} & =2-2 \cos \theta
\end{aligned}
$$

and

$$
1+\rho^{2}-2 \rho \cos \theta-(2-2 \cos \theta)=(\rho-1)(\rho+1-2 \cos \theta) \geq 0 .
$$


As $[\mu]_{B}$ is an infinitesimal non-Strebel point, $\left\|\left.\mu\right|_{R \backslash E}\right\|_{\infty}=k_{0} \geq\left\|\left.\mu\right|_{E}\right\|_{\infty}$, so by (2.5)-(2.8),

$$
\left\|t_{1} \mu_{t_{1}}-t_{2} \mu_{t_{2}}\right\|_{\infty}=\left\|\left.\frac{t_{1}-t_{2}}{k_{0}} \mu(z)\right|_{R \backslash E}\right\|_{\infty}=\left|t_{1}-t_{2}\right| .
$$

Also because $[\mu]_{B}$ is an infinitesimal non-Strebel point, $\mu$ has a degenerate Hamilton sequence $\left\{\varphi_{n}\right\}\left(\left\{\varphi_{n}\right\}\right.$ is called degenerate if $\varphi_{n} \rightarrow 0$ locally uniformly in $R$ ). Hence

$$
\begin{aligned}
\lim _{n \rightarrow \infty}\left|\int_{R}\left(t_{1} \mu_{t_{1}}-t_{2} \mu_{t_{2}}\right) \varphi_{n}\right| & =\lim _{n \rightarrow \infty}\left|\int_{R \backslash E} \frac{t_{1}-t_{2}}{k_{0}} \mu \varphi_{n}+\int_{E}\left(\frac{t_{1}}{\left|t_{1}\right|}-\frac{t_{2}}{\left|t_{2}\right|}\right) \mu \varphi_{n}\right| \\
& =\left|\frac{t_{1}-t_{2}}{k_{0}}\right| \lim _{n \rightarrow \infty}\left|\int_{R \backslash E} \mu \varphi_{n}\right|=\left|t_{1}-t_{2}\right| .
\end{aligned}
$$

From (2.4), (2.5), (2.9) and (2.10), we conclude that

$$
d_{B}\left(\left[t_{1} \mu_{t_{1}}\right]_{B},\left[t_{2} \mu_{t_{2}}\right]_{B}\right)=\left|t_{1}-t_{2}\right| .
$$

(3) Suppose $\left|t_{1}\right| \leq k_{0}$ and $\left|t_{2}\right| \geq k_{0}$ (the case $\left|t_{1}\right| \geq k_{0}$ and $\left|t_{2}\right| \leq k_{0}$ can be addressed similarly). In this case, by the definition of $\mu_{t}(z)$,

$$
d_{B}\left(\left[t_{1} \mu_{t_{1}}\right]_{B},\left[t_{2} \mu_{t_{2}}\right]_{B}\right)=\inf _{\eta \in\left[t_{1} \mu_{t_{1}}-t_{2} \mu_{t_{2}}\right]_{B}}\|\eta\|_{\infty}
$$

where

$$
t_{1} \mu_{t_{1}}-t_{2} \mu_{t_{2}}=\left\{\begin{array}{l}
\frac{t_{1}-t_{2}}{k_{0}} \mu(z) \quad \text { for } z \in R \backslash E, \\
\left(\frac{t_{1}}{k_{0}}-\frac{t_{2}}{\left|t_{2}\right|}\right) \mu(z) \quad \text { for } z \in E .
\end{array}\right.
$$

By a similar argument as in case (2), we can draw the conclusion that

$$
d_{B}\left(\left[t_{1} \mu_{t_{1}}\right]_{B},\left[t_{2} \mu_{t_{2}}\right]_{B}\right)=\left|t_{1}-t_{2}\right| \text {. }
$$

So, by establishing (2.3), (2.11) and (2.12), we have completed the proof of the lemma.

Proofs of THEOREMS 2.1 AND 2.2. We take a sequence $\left\{E_{n}: n=1,2, \ldots\right\}$ of compact subsets of $R$, such that $E_{n} \subset E_{n+1}$ for each $n=1,2, \ldots$ and $R=\bigcup_{n=1}^{\infty} E_{n}$. For each $n$, we define a Beltrami differential $\mu_{t}^{(n)}$ by (2.1) and (2.2). Let $\Gamma_{n}: \triangle \rightarrow$ $B(R)$ be the map $t \mapsto\left[t \mu_{t}^{(n)}\right]_{B}$. Then, from Lemma 2.4, we know that $\Gamma_{n}$ is an isometry with

$$
\Gamma_{n}(0)=[0]_{B} \quad \text { and } \quad \Gamma_{n}\left(\|\mu\|_{\infty}\right)=[\mu]_{B} \quad \text { for } n=1,2, \ldots
$$

Now we construct a subsequence $\left\{\Gamma_{n_{j}}\right\}$ of $\left\{\Gamma_{n}\right\}$ such that $\Gamma_{n_{j}}(\triangle) \neq \Gamma_{n_{k}}(\triangle)$ whenever $n_{j} \neq n_{k}$. We start with $\Gamma_{1}$ as $\Gamma_{n_{1}}$ and fix a real number $t_{0} \in\left(k_{0}, 1\right)$. For $n>n_{1}$, by definition,

$$
d_{B}\left(\Gamma_{1}\left(t_{0}\right), \Gamma_{n}\left(t_{0}\right)\right)=\inf _{\eta \in\left[t_{0} \mu_{t_{0}}^{(1)}-t_{0} \mu_{t_{0}}^{(n)}\right]_{B}}\|\eta\|_{\infty}
$$


where

$$
t_{0} \mu_{t_{0}}^{(1)}(z)-t_{0} \mu_{t_{0}}^{(n)}(z)=\left\{\begin{array}{l}
0 \quad \text { for } z \in R \backslash E_{n}, \\
0 \text { for } z \in E_{1}, \\
\left(\frac{t_{0}}{k_{0}}-\frac{t_{0}}{\left|t_{0}\right|}\right) \mu(z) \quad \text { for } z \in E_{n} \backslash E_{1} .
\end{array}\right.
$$

Set $\varepsilon=\left(k_{0} / 4\right)>0$. As $\left\{\varphi_{n}\right\}$ is a degenerate Hamilton sequence of $\mu$, we can choose $\varphi_{m} \in\left\{\varphi_{n}\right\}$ such that

$$
\int_{E_{1}}\left|\varphi_{m}\right|<\frac{1}{4}
$$

and

$$
\left|\int_{R} \mu \varphi_{m}\right|>k_{0}-\varepsilon .
$$

Since $R=\bigcup_{n=1}^{\infty} E_{n}$, for the given $\varphi_{m}$ there is a number $n_{2}$ such that for any $n \geq n_{2}$,

$$
\int_{R \backslash E_{n}}\left|\varphi_{m}\right|<\frac{1}{4} .
$$

By (2.15)-(2.17), when $n \geq n_{2}$,

$$
\begin{aligned}
\left|\int_{R}\left(t_{0} \mu_{t_{0}}^{(1)}-t_{0} \mu_{t_{0}}^{(n)}\right) \varphi_{m}\right| & =\left|\int_{E_{n} \backslash E_{1}}\left(\frac{t_{0}}{k_{0}}-\frac{t_{0}}{\left|t_{0}\right|}\right) \mu \varphi_{m}\right| \\
& \geq\left(\frac{t_{0}}{k_{0}}-\frac{t_{0}}{\left|t_{0}\right|}\right)\left[\left|\int_{R} \mu \varphi_{m}\right|-\left|\int_{E_{1}} \mu \varphi_{m}\right|-\left|\int_{R \backslash E_{n}} \mu \varphi_{m}\right|\right] \\
& \geq\left(\frac{t_{0}}{k_{0}}-\frac{t_{0}}{\left|t_{0}\right|}\right)\left[k_{0}-\varepsilon-\frac{k_{0}}{4}-\frac{k_{0}}{4}\right] \\
& =\left(\frac{t_{0}}{k_{0}}-\frac{t_{0}}{\left|t_{0}\right|}\right) \frac{k_{0}}{4}>0 .
\end{aligned}
$$

Now, (2.13) and (2.18) imply that $d_{B}\left(\Gamma_{n_{1}}\left(t_{0}\right), \Gamma_{n}\left(t_{0}\right)\right)>0$ when $n \geq n_{2}$, so we have found $n_{2}$ such that $\Gamma_{n}\left(t_{0}\right) \neq \Gamma_{n_{1}}\left(t_{0}\right)$ whenever $n \geq n_{2}$. A similar discussion yields a number $n_{3}>n_{2}$ such that $\Gamma_{n}\left(t_{0}\right) \neq \Gamma_{n_{2}}\left(t_{0}\right)$ whenever $n \geq n_{3}$. Repeating the same argument, we obtain a sequence $n_{j}$ such that $\Gamma_{n_{j}}\left(t_{0}\right) \neq \Gamma_{n_{k}}\left(t_{0}\right)$ whenever $n_{j} \neq n_{k}$.

On the other hand, $\Gamma_{n}(\triangle)=\Gamma_{m}(\triangle)$ implies $\Gamma_{n}\left(t_{0}\right)=\Gamma_{m}\left(t_{0}\right)$. If $\tilde{t_{0}}$ is a point in $\triangle$ such that $\Gamma_{n}\left(t_{0}\right)=\Gamma_{m}\left(\widetilde{t_{0}}\right)$, then for each $t \leq k_{0}$ the isometric property of $\Gamma$ and (2.1) give

$$
\begin{aligned}
\left|t_{0}-t\right| & =d_{B}\left(\Gamma_{n}\left(t_{0}\right), \Gamma_{n}(t)\right) \\
& =d_{B}\left(\Gamma_{m}\left(\widetilde{t_{0}}\right), \Gamma_{n}(t)\right)=d_{B}\left(\Gamma_{m}\left(\widetilde{t_{0}}\right), \Gamma_{m}(t)\right) \\
& =\left|\widetilde{t_{0}}-t\right| .
\end{aligned}
$$

Since $\left|t_{0}-t\right|=\left|\tilde{t_{0}}-t\right|$ for every $t \leq k_{0}$, we have $t_{0}=\tilde{t_{0}}$.

Thus we have constructed a subsequence $\left\{\Gamma_{n_{j}}\right\}$ such that $\Gamma_{n_{j}}(\triangle) \neq \Gamma_{n_{k}}(\triangle)$ whenever $n_{j} \neq n_{k}$. This completes the proof. 


\section{Hamilton sequences for extremal Beltrami differentials}

First we recall the following known result.

THEOREM A. Suppose that $\mu \in M(R)$ is extremal in Teichmüller space $T(R)$ and that $\left\{\varphi_{n}\right\}$ is a sequence in $A_{1}(R)$. If, for some sequence $\left\{k_{n}\right\},\left[k_{n}\left(\overline{\varphi_{n}} /\left|\varphi_{n}\right|\right)\right]$ converges in the Teichmüller metric to $[\mu]$, then $\left\{\varphi_{n}\right\}$ is a Hamilton sequence for $\mu$.

Theorem A was proved by Gardiner [3] (see also [6, 7]). In this note, we shall consider the corresponding problem in infinitesimal Teichmüller space and prove the following result.

THEOREM 3.1. Suppose that $\mu \in M(R)$ is extremal in infinitesimal Teichmüller space $B(R)$ and that $\left\{\varphi_{n}\right\}$ is a sequence in $A_{1}(R)$. If, for some sequence $\left\{k_{n}\right\},\left[k_{n}\left(\bar{\varphi}_{n} /\left|\varphi_{n}\right|\right)\right]_{B}$ converges in the infinitesimal Teichmüller metric to $[\mu]_{B}$, then $\left\{\varphi_{n}\right\}$ is a Hamilton sequence for $\mu$.

REMARK 3.2. Since the condition that $\left[\mu_{n}\right]_{B}$ be convergent in the infinitesimal Teichmüller metric to $[\mu]_{B}$ does not imply that $\left[\mu_{n}\right]$ will converge in the Teichmüller metric to $[\mu]$, and vice versa, Theorem 3.1 cannot be directly deduced from Theorem A. Shen [10] gave a counterexample which shows that the converse of Theorem A is not true. It is easy to see that the counterexample in [10] is also suitable for showing that the converse of Theorem 3.1 does not hold.

To prove Theorem 3.1, the following 'infinitesimal main inequality' is needed.

Theorem B [1]. Suppose $\mu, v \in M(R)$ and $\mu \approx \nu$; then

$$
\begin{aligned}
& \int_{R}|\varphi|\left(1-|\mu|^{2}\right) \leq \int_{R}|\varphi|\left|1-\mu \frac{\varphi}{|\varphi|}\right|^{2}\left(\left|1+\nu \frac{\varphi}{|\varphi|} \frac{1-\bar{\mu}(\bar{\varphi} /|\varphi|)}{1-\mu(\varphi /|\varphi|)}\right|^{2}\right)\left(1-|\nu|^{2}\right)^{-1}, \\
& \quad \text { for all } \varphi \in A(R) .
\end{aligned}
$$

LEMMA 3.3. Let $\mu \in M(R)$; then

$$
-\|\mu\| \leq \frac{\int_{R}|\mu|^{2}|\varphi|-\operatorname{Re} \int_{R} \mu \varphi}{1-\operatorname{Re} \int_{R} \mu \varphi} .
$$

PROOF. For any $v \approx \mu$,

$$
\left|1+v \frac{\varphi}{|\varphi|} \frac{1-\bar{\mu}(\bar{\varphi} /|\varphi|)}{1-\mu(\varphi /|\varphi|)}\right|^{2}\left(1-|\nu|^{2}\right)^{-1} \leq \frac{(1+|\nu|)^{2}}{1-|\nu|^{2}} .
$$

From (3.1) and (3.3),

$$
\frac{1+\|\mu\|}{1-\|\mu\|} \geq \frac{\int_{R}|\varphi|\left(1-|\mu|^{2}\right)}{\int_{R}|\varphi||1-\mu(\varphi /|\varphi|)|^{2}},
$$

and then (3.2) can be easily deduced from (3.4). 
LeMma 3.4. Let $[\mu]_{B}$ and $[v]_{B}$ be two points in $B(R)$, and let $t>0$ be a given number. Then

$$
d_{B}\left([\mu]_{B},[\nu]_{B}\right)=t d_{B}\left(\left[\frac{\mu}{t}\right]_{B},\left[\frac{v}{t}\right]_{B}\right) .
$$

PROOF. If $\eta \in[\mu-v]_{B}$ and $\eta$ is extremal, it is easy to show that $(\eta / t) \in[(\mu-v) / t]_{B}$ and that $(\eta / t)$ is extremal. Then (3.5) can be derived from the definition of the infinitesimal Teichmüller distance.

Proof of Theorem 3.1. We write $k=\|\mu\|$. Since $d_{B}\left(\left[k_{n}\left(\bar{\varphi}_{n} /\left|\varphi_{n}\right|\right)\right]_{B},[\mu]_{B}\right) \rightarrow 0$, we have $k_{n} \rightarrow k$. By Lemma 3.4, without loss of generality we can assume $k<\frac{1}{2}$ and $k_{n}<\frac{1}{2}$. Under these assumptions, we use Lemma 3.3 with $k_{n}\left(\bar{\varphi}_{n} /\left|\varphi_{n}\right|\right)-\mu$ and $\varphi=\varphi_{n}$ to get

$$
\begin{aligned}
-\left\|k_{n} \frac{\bar{\varphi}_{n}}{\left|\varphi_{n}\right|}-\mu\right\| & \leq \frac{\int_{R}\left|k_{n}\left(\bar{\varphi}_{n} /\left|\varphi_{n}\right|\right)-\mu\right|^{2}\left|\varphi_{n}\right|-\operatorname{Re} \int_{R}\left(k_{n}\left(\bar{\varphi}_{n} /\left|\varphi_{n}\right|\right)-\mu\right) \varphi_{n}}{1-\operatorname{Re} \int_{R}\left(k_{n}\left(\bar{\varphi}_{n} /\left|\varphi_{n}\right|\right)-\mu\right) \varphi_{n}} \\
& =\frac{k_{n}^{2}-k_{n}+\operatorname{Re} \int_{R}\left[|\mu|^{2}\left|\varphi_{n}\right|+\left(1-2 k_{n}\right) \mu \varphi_{n}\right]}{1-k_{n}+\operatorname{Re} \int_{R} \mu \varphi_{n}} \\
& =-k_{n}+\frac{\operatorname{Re} \int_{R}\left[|\mu|^{2}\left|\varphi_{n}\right|+\left(1-k_{n}\right) \mu \varphi_{n}\right]}{1-k_{n}+\operatorname{Re} \int_{R} \mu \varphi_{n}} .
\end{aligned}
$$

From $d_{B}\left([0]_{B},\left[k_{n} \bar{\varphi}_{n} /\left|\varphi_{n}\right|\right]_{B}\right)=k_{n}$ and

$$
d_{B}\left([\mu]_{B},\left[k_{n} \frac{\bar{\varphi}_{n}}{\left|\varphi_{n}\right|}\right]_{B}\right)=\left\|k_{n} \frac{\bar{\varphi}_{n}}{\left|\varphi_{n}\right|}-\mu\right\|
$$

it follows that

$$
\begin{aligned}
& d_{B}\left([0]_{B},\left[k_{n} \frac{\bar{\varphi}_{n}}{\left|\varphi_{n}\right|}\right]_{B}\right)-d_{B}\left([\mu]_{B},\left[k_{n} \frac{\bar{\varphi}_{n}}{\left|\varphi_{n}\right|}\right]_{B}\right) \\
& \quad \leq \frac{\operatorname{Re} \int_{R}\left[|\mu|^{2}\left|\varphi_{n}\right|+\left(1-k_{n}\right) \mu \varphi_{n}\right]}{1-k_{n}+\operatorname{Re} \int_{R} \mu \varphi_{n}} .
\end{aligned}
$$

Now we prove that

$$
\operatorname{Re} \int_{R} \mu \varphi_{n} \rightarrow\|\mu\|=k .
$$

Suppose (3.8) is not true; then there must be a subsequence of $\left\{\varphi_{n}\right\}$, which we also denote by $\left\{\varphi_{n}\right\}$, such that

$$
\operatorname{Re} \int_{R} \mu \varphi_{n} \rightarrow \widetilde{k}<k<\frac{1}{2} .
$$

Because $d_{B}\left([0]_{B},\left[k_{n} \bar{\varphi}_{n} /\left|\varphi_{n}\right|\right]_{B}\right)=k_{n} \rightarrow k$ and $d_{B}\left([\mu]_{B},\left[k_{n} \bar{\varphi}_{n} /\left|\varphi_{n}\right|\right]_{B}\right) \rightarrow 0$ upon taking $n \rightarrow \infty$, by (3.7) we conclude that

$$
k \leq \frac{k^{2}+(1-k) \tilde{k}}{1-k+\widetilde{k}} .
$$


By direct computation, we find that

$$
(2 k-1) \tilde{k} \leq(2 k-1) k .
$$

Since $k<\frac{1}{2}$, it follows that $\widetilde{k} \geq k$, which contradicts (3.9). Therefore (3.7) holds and $\left\{\varphi_{n}\right\}$ is a Hamilton sequence for $\mu$. This completes the proof.

\section{Acknowledgement}

The authors would like to thank the referees for valuable suggestions and comments which improved the quality of the paper.

\section{References}

[1] V. Božin, N. Lakic, V. Marković and M. Mateljević, 'Unique extremality', J. Anal. Math. 75 (1998), 299-338.

[2] J. Fan and J. Chen, 'On the equivalence of extremal Teichmüller mapping', Sci. China. Ser. A 51 (2008), preprint

[3] F. P. Gardiner, 'Approximation of infinite dimension Teichmüller spaces', Trans. Amer. Math. Soc. 282 (1984), 367-383.

[4] Teichmüller Theory and Quadratic Differentials (Wiley-Interscience, New York, 1987).

[5] F. P. Gardiner and N. Lakic, Quasiconformal Teichmüller Theory, Mathematical Surveys and Monographs, 76 (American Mathematical Society, Providence, RI, 2000).

[6] N. Lakic, 'Strebel points', Contemp. Math. 211 (1997), 417-431.

[7] Z. Li, 'Strebel differentials and Hamilton sequences', Sci. China Ser. A 44 (2001), 969-979.

[8] - 'Geodesics disks in Teichmüller space', Sci. China Ser. A 48 (2005), 1075-1082.

[9] Y. Shen, 'On Teichmüller geometry', Complex Var. Theory Appl. 44 (2001), 73-83.

[10] , 'A note on Hamilton sequences for extremal Beltrami coefficients', Proc. Amer. Math. Soc. 129 (2001), 105-109.

[11] G. Yao, 'Is there always an extremal Teichmüller mapping', J. Anal. Math. 94 (2004), 363-375.

\section{FAN JINHUA, Department of Applied Mathematics,}

Nanjing University of Science and Technology, Nanjing, 210094,

People's Republic of China

e-mail: jinhuafan@hotmail.com

CHEN JIXIU, School of Mathematical Sciences, Fudan University, Shanghai, 200433, People's Republic of China 